\title{
Pedagogías críticas y educación en derechos humanos Una mirada desde escenarios escolares y no escolares
}

CRITICAL PEDAGOGIES AND HUMAN RIGHTS EDUCATION.
A VIEW FROM SCHOOL AND NONSCHOOL SETTINGS

PEDAGOGIAS CRÍTICAS E EDUCAÇÃO EN DIREITOS HUMANOS:

UM OLHAR DESDE OS CENÁRIOS ESCOLARES E NÃO ESCOLARES

Oscar Orlando Espinel Bernal ** / bespinelo@yahoo.es

\section{Resumen}

La formación en derechos humanos no puede reducirse a su reconocimiento y exigibilidad, sino que ha de avanzar hacia la consolidación de una cultura de derechos humanos. El análisis comparativo basado en el análisis documental y en las prácticas de dos proyectos en escenarios formales y no formales, permite establecer que la constitución de subjetividades políticas desde la perspectiva de los derechos humanos no solo se ve fortalecida por las propuestas freireanas, sino que a su vez, ofrece un nicho dentro del cual las pedagogías críticas pueden repensarse y concretarse.

\section{Summary}

Human Rights education cannot be reduced to its recognition and demand. It must move towards the consolidation of a culture on Human Rights. The comparative analysis based on the documentary analysis and the practices of two projects in formal and informal settings, allows to establish that the constitution of political subjectivities from the perspective of Human Rights is not only reinforced by the Freirean proposals; they in turn, provide a niche where critical pedagogy can be rethought and become concrete.

\section{Resumo}

A formação em direitos humanos não pode se reduzir ao seu reconhecimento e exigibilidade; porém, tem de avançar para a consolidação de uma cultura de direitos humanos. A análise comparativa, baseada na análise documental e nas práticas dos projetos em cenários formais e não formais, permite de estabelecer que a constituição de subjetividades políticas desde a perspectiva dos direitos humanos não só se vê fortalecida pelas propostas freireanas, senão que a sua vez oferecem um nicho dentro do qual as pedagogias críticas podem se repensar e se concretizar.
Palabras clave

Educación emancipadora, diálogo de saberes, sujetos crítico-políticos, subjetividades políticas, derechos humanos.

Key words

Emancipating education, dialog of knowledge, critical-political subjects, political subjectivities, Human Rights.

Palavras chave

Emancipatório educação, diálogo de conhecimento, crítica e sujeitos políticos, subjetividades políticas, direitos humanos.

\footnotetext{
Artículo presentado a la revista Nodos y Nudos el 14 de agosto de 2009 asistiendo a la convocatoria para su publicación número 27.

Este artículo recoge los principales conceptos y propuestas de nuiestro trabajo de tesis para la Especialización en Pedagogía de la Universidad Pedagógica Nacional titulada "Aportes de las pedagogías críticas inspiradas en la obra de Paulo Freire, a la constitución de subjetividades en torno a una cultura de derechos humanos. Análisis de dos experiencias", investigación llevada a cabo en el 2008 en torno a escenarios escolares y no escolares.

** Docente del Colegio Cafam, estudiante de la Maestría en Educación de la Universidad Pedagógica Nacional y Especialista en Pedagogía del mismo centro universitario.
} 
El problema que inspira y orienta este trabajo investigativo realizado como trabajo de grado dentro de la Especialización en Pedagogía de la Universidad Pedagógica, consiste en establecer los aportes que podrían hacer las pedagogías críticas desde la obra de Paulo Freire a una educación en derechos humanos. En relación con estos referentes, la educación, dentro de este proyecto investigativo, no se entiende como un ejercicio cuyo limitado propósito sea el memorizar y conocer el catálogo de los derechos reconocidos internacionalmente y protegidos desde las constituciones de cada país. Desde ya se empieza a vislumbrar un nexo especial con la obra freireana en donde el acto educativo supera estas prácticas reproduccionistas, problematizando y abordando críticamente sus objetos de estudio. Objetos de estudio que se construyen desde las mismas vivencias, problemáticas y realidades de los sujetos cognoscentes. No son solo constructos teóricos y abstractos, sino que toman vida en la vida misma de los participantes, en su relación directa con el contexto. El conocimiento no es algo heredado, ni algo que se transmita de una conciencia a otra; por el contrario, el conocimiento es una construcción llevada a cabo por sujetos cognoscentes y con conciencia de sí mismos, de su realidad, de su relación con otros.

Desde esta perspectiva, la formación en derechos humanos se aleja de las prácticas transmisionistas pues dichas prácticas promueven el ejercicio memorístico de conceptos e ideas que difícilmente lograrán trascender el cuaderno. Los derechos humanos no son solo cláusulas teóricas o legalistas, sino que son, en sí mismos, principios que se hacen presentes en las relaciones humanas. Por esta razón, no se quedan en un aula, o en un libro ni en una constitución, porque cada vez que me relaciono lo hago como ser humano, como persona con dignidad. Los derechos humanos inundan toda relación social, impregnan a todos los sujetos en su existencia.

En consecuencia, la formación en derechos humanos no se queda en el aprenderlos y reconocerlos, sino en fundar e impulsar las posibilidades para la consolidación de una cultura de derechos humanos que inspire y abarque las relaciones humanas.

\section{Las pedagogías críticas en el campo de la formación en derechos humanos}

La metodología que se empleó en este trabajo investigativo está orientada desde la perspectiva del enfoque cualitativo. Pretende abordar las prácticas educativas y pedagógicas de las experiencias educativas consultadas desde sus contextos y sus relatos con el objeto de obtener y organizar la información1. En este enfoque investigativo no se trata de confirmar hipótesis o comprobar teorías; por el contrario, se trata de dejar hablar a las experiencias y observar los fenómenos y situaciones que emergen.

Las entrevistas fueron aplicadas en dos experiencias de educación en derechos humanos realizadas en diferentes localidades de Bogotá. Una de ellas fue liderada por la Fundación Codiepsir2 (Corporación Escuela Simón Rodríguez) en la localidad de Ciudad Bolívar y la Escuela de Derechos Humanos, Paz y Convivencia (EDHPC)3.

A partir de este análisis comparativo de las prácticas develadas en los discursos de los sujetos entrevistados, fuimos constatando la presencia de categorías que denominamos emergentes. Estas "categorías emergentes" permiten unificar de alguna manera las prácticas indagadas y construir algunas conclusiones relacionadas con la intención de este trabajo investigativo. A continuación presentamos algunos aspectos del análisis final de las categorías teóricas en relación con las categorías emergentes de la matriz comparativa de resultados de las entrevistas.

1 El instrumento empleado es una entrevista abierta en medio de una dinámica dialógica con la intención de extraer la mayor cantidad de información a partir de las experiencias del actor entrevistado. "Esto implica que la información ha sido experimentada y absorbida por el entrevistado. Orientación, deformación o interpretación que muchas veces resulta más interesante, informativamente, que la propia exposición cronológica o sistemática de acontecimientos más o menos factuales" (Delgado y Gutiérrez 1999, cap. 8).

2 Desde el ámbito no formal trabaja con la comunidad de la localidad de Ciudad Bolívar en espacios de concienciación, fortalecimiento de procesos de formación de sujetos políticos y desarrollo de alternativas para el mejoramiento de las condiciones de las poblaciones empobrecidas a partir del acompañamiento y la formación. Ver contextualización en los documentos anexos del Trabajo de Tesis.

3 Este es un proyecto del Centro de Investigación y Educación Popular -CINEP- que tiene por objeto aportar a la construcción de una cultura de la paz, los derechos humanos y de una sociedad más justa; al fortalecimiento de procesos, organizaciones y redes sociales a través de la formación, reflexión y acompañamiento a procesos de formación desde la perspectiva de los derechos humanos. Este proyecto se llevó a cabo en instituciones educativas del distrito de las localidades de Bosa y Tunjuelito. Ver en los documentos anexos del Trabajo de Tesis la contextualización y la entrevista realizada a uno de sus integrantes. 


\section{Formación ético-política desde la perspectiva de la educación en derechos humanos}

Toda práctica educativa es una práctica política ${ }^{4}$ ya sea legitimando las prácticas sociales, imaginarios e idearios vigentes en la sociedad de donde emerge o, planteando y transformando la realidad social y sus estructuras con la constitución de esos nuevos sentidos, significados, posiciones, prácticas, valores y utopías que se instauran como renovación de aquellos que tenían la soberanía sobre la cultura, la política y la sociedad. Además, esta práctica educativa política involucra una ética5, unos referentes frente a lo bueno y lo malo, a lo justo y lo injusto, a lo que nos hace humanos y lo que nos deshumaniza. Desde allí se plantean las utopías, los sueños, la transformación o la reproducción.

Es así como la propuesta freireana es una propuesta pedagógica eminentemente ético-política que entiende el proceso educativo como un proceso que conduce a una lectura crítica de la realidad, que fortalece o posibilita el afloramiento de la conciencia crítica. Un proceso que devuelve la palabra a los silenciados, junto con su facultad de pronunciar y recrear el mundo. La educación empodera a los históricamente "oprimidos" y alimenta la esperanza necesaria para emprender el camino hacia el cambio social y la construcción de una cultura en donde los derechos humanos sean el soporte de las relaciones sociales. La educación es una acción cultural emancipadora.

En las prácticas educativas examinadas 6 la formación se inicia con un reconocimiento de la realidad social y política de nuestro país a partir de las vivencias personales y barriales al interior de las mismas comunidades. Este es un trabajo de concienciación, que desde ya está ligado con la politización de los sujetos, propósito fundamental para las pedagogías críticas. Politización

4 La obra de Freire insiste constantemente en esta idea: "Y es, precisamente su politicidad, su imposibilidad de ser neutra, lo que exige del educador o de la educadora su eticidad" (Freire, 2007, p. 74). Ver también Torres (2007, p. 31).

5 Educar es formar éticamente, no sólo transmitir conocimientos. Ver Freire (1997, p. 34).

6 Magendzo (2005) nos propone algunas ideas que nos hablan del sentido y significado de la educación en derechos humanos y, a su vez, Torres (2007), nos plantea un núcleo común de elementos de la educación popular. Estos elementos nos permiten analizar e identificar los proyectos educativos trabajados haciendo un paralelo con los sentidos allí expuestos de la educación en derechos humanos. que compromete al sujeto con la iniciación de procesos de transformación que surge desde la preocupación por las mismas problemáticas de su comunidad ante la constatación de que son reales y además, injustas, indignas y en muchas ocasiones inhumanas.

Es evidenciar que el estado no es el gobierno en cabeza del presidente que se aloja en la Casa de Nariño, sino que el estado es la misma comunidad7, que somos parte del estado y que las problemáticas de las comunidades nos competen, porque también somos sujetos de deliberación y acción frente a las situaciones de nuestra comunidad. Es una dinámica que busca romper con la indiferencia y con las prácticas de delegar el problema y las responsabilidades en otros ante la idea de que se carece de competencia para afrontar el problema. Es necesario romper con posturas conformistas y con aquellas que se quedan en la denuncia, despertando el sentido de pertenencia y compromiso que den lugar a la acción y al emprendimiento de procesos por las comunidades.

Es entender que los asuntos de la comunidad son asuntos de todos y no sólo de las autoridades y de las personas que son víctimas y victimarios, porque la situación nos involucra a todos. Comprometen nuestra vida pues es la vida misma la que se encuentra en juego. "Y a medida que se va avanzando, a medida que se va metiendo en el cuento, que no es de derechos humanos sino que es en relación con lo público, es pensarnos en colectivo" (E, párrafo 66). De esa manera, a partir de esos procesos de concienciación se inician acciones como son las de exigibilidad, acciones de tutela, apoyo a movimientos acciones populares, organizaciones comunitarias frente a alguna situación.

El propósito de estos proyectos no es adoctrinar, sino, incluso, problematizar los mismos discursos que se hacen circular al interior del aula para garantizar el ejercicio del pensamiento crítico y además, propiciar la obtención de procesos de aprendizaje más significativos e interiorizados por los sujetos en franca resistencia frente a las prácticas memorísticas. "La escuela siempre va a decir que tenemos que respetarnos, el problema es que no dice por qué hay que respetarnos. Es como un principio 'hay que respetar al otro' y ya" (E, párrafo 75). Así, en palabras de Codiepsir, en estos proyectos se está "construyendo pedagogía"; pues la pedagogía

\footnotetext{
7 En la referencia a los relatos recogidos en las entrevistas realizadas, se respetará la codificación empleada en el Trabajo de Tesis. Ver E, párrafo 64.
} 
y el acto educativo mismo, no son algo ya terminado, sino por el contrario, están en constante re-creación y actualización acorde con las necesidades e intereses de los participantes y apoyados en el diálogo y la interacción entre docentes y educandos.

No se trata de hablar desde los libros ni los informes, ni mucho menos refugiados en un aula en un aislamiento absurdo frente a la realidad. Se trata de una pedagogía de la vida. Una pedagogía donde la vida es el principal motor, el eje y el centro de la reflexión. No se trata de hablar de cosas hipotéticas, abstractas, ni siquiera desde el "deber ser", sino desde la vida misma y por la vida misma. Por el derecho a vivir dignamente y existir en condiciones dignas, humanas.

\section{Entonces, más que partir de un énfasis teórico de qué son los derechos humanos, que no se deja de lado, se parte de: pensemos cómo nosotros convivimos diariamente y si algo de eso se pare- ce a los derechos humanos, pues empecemos a relacionarlos. Y nos dábamos cuenta que muchas de las cosas que nosotros hacemos diariamente tienen que ver con los derechos humanos. Es más, hasta prácticas familiares, prácticas muy privadas, tienen que ver con derechos humanos (E, párrafo 10).}

Es partir de la vida para darle validez a las teorías, para dar sentido a los discursos teóricos, académicos y conceptuales. "Y a reflexionar sobre la vida desde los derechos. La importancia que tiene la vida para hablar de derechos, porque hablar de los derechos en vacío, sin tocar la problemática que a ellos les afecta, pues no tendría sentido" (C1, párrafo 31). Por eso, es imprescindible partir de ese diálogo de saberes, donde estos discursos y estas prácticas se insertan, emergen y se reflejan en las acciones diarias, en las vivencias, experiencias. Para una cultura de derechos humanos, lo primero es encontrar que éstos no son un invento sino parte fundamental y esencial de la vida y de las relaciones intersubjetivas. No son un privilegio, ni un regalo, ni una concesión, son precisamente eso, un derecho: pero no un derecho otorgado, sino un derecho natural, propio, del cual somos partícipes por el hecho de ser humanos 8 .

8 "Los derechos humanos tienden a garantizar aquella clase de bienes a los que las personas no estarían dispuestos a renunciar, puesto que esa renuncia significaría lo mismo que un abandono de su condición de humanos" (Defensoría del Pueblo, 2004, p. 22).

\section{Constitución de subjetividades}

La realidad es un continuo, como afirmara Zemelman (2006, p. 22), entre dos extremos, uno de ellos el individuo y el otro la sociedad; en su interacción, en la tensión constante que los dinamiza, surgen las subjetividades que se encarnan en las entrañas del sujeto y lo constituyen como sujeto social. Aunque el hombre se encuentra en medio de un contexto que lo inserta en una cultura desde donde sus prácticas adquieren sentido, el sujeto es también artífice de esos sentidos; de tal manera que en sus prácticas valida esos sentidos o los transforma creando nuevas realidades, nuevos mundos, nuevos sujetos.

La transformación se inicia y se fortalece en el proceso de involucrar y afectar la subjetividad. Una cultura de derechos humanos requiere de una constitución de subjetividades frente al mundo de los derechos humanos. No es suficiente con lograr estados de conciencia frente a las realidades contradictorias y opresoras de las estructuras sociales vigentes y de sus prácticas sociales injustas y deshumanizantes. Es necesario construir nuevos sentidos, nuevos significados que incidan en las prácticas sociales y que permitan su revaloración y eventual transformación. Este estado no se alcanza con la simple denuncia ni con el discurso racional, se requiere afectar el mundo de la subjetividad y jalonar procesos de resignificación.

La subjetividad se constituye y expresa en el mundo de la cultura; un mundo simbólico fuente de sentidos y significados frente a la realidad y a la vida humana. En consecuencia, la subjetividad es de naturaleza simbólica (Torres, 2006) y desborda los límites del discurso y de la racionalidad. Desde la subjetividad se construyen los sentidos desde los cuales se entiende el mundo, se pronuncia y se actúa. Es allí donde se inscribe la acción cultural de las prácticas educativas. "La alfabetización crítica se sitúa, según Freire, en la intersección entre el lenguaje (particular forma de producción cultural), la cultura (formas ideológicas en las que un grupo social vive sus circunstancias y condiciones de vida y les confiere sentido), el poder (el ejercicio de pronunciarse y transformar la realidad) y la historia (como lo que está siendo y dándose)" (El Achkar, 2002, p. 5). Si afirmamos que las prácticas humanas se encuentran en un mundo histórico y cultural, estamos comprendiendo al ser humano como sujeto social pues la cultura no es un producto individual sino resultado y condición de la intersubjetividad. 
Toda subjetividad se torna intersubjetiva. En el individuo se hacen presentes otras subjetividades que impregnan y constituyen la propia subjetividad. De esta manera, encontramos otra característica de la subjetividad, y es su carácter vinculante. A través de la subjetividad el sujeto construye un vínculo con los demás, se relaciona con los otros sujetos. En esta relación logra construir su identidad y se circunscribe en el mundo cultural de la sociedad.

\section{Educación desde y para lo comunitario}

\section{Y creo que lo importante de la formación en derechos humanos está basado un poco más en el ejercicio de acciones en derechos humanos desde lo cotidiano y desde la forma en que ellos se van relacionando con otros jóvenes, con la familia, con los amigos y en la forma en que se asuman esas prácticas (C1, párrafo 61).}

El pensamiento crítico, como ya se ha expuesto anteriormente, no solo implica un estado de conciencia frente a las contradicciones e ideologías dominantes que falsean la realidad; también va acompañado de la acción, de una acción transformadora. El pensamiento crítico es un ejercicio que se realiza a partir del asumirse como ser creador y no simplemente repetidor o perpetuador de las ideas que circulan. "Por eso, alcanzar la comprensión más crítica de la situación de opresión todavía no libera a los oprimidos. Sin embargo, al desnudarla, dan un paso para superarla, siempre que se empeñen en la lucha política por la transformación de las condiciones concretas en que se da la opresión" (Freire, 2007, p. 29).

En el ámbito de los derechos humanos suele presentarse la situación según la cual se intenta transmitir un consolidado, quizás teórico, e incluso, moralista, que acerca los derechos a un catálogo de normas y principios ya dados y que deben cumplirse. Nada más equivocado que esta concepción. Los derechos humanos son un proyecto inacabado, en constante actualización y validación en las mismas prácticas. Por esta razón, más que recitar una serie de principios se trata de lograr una comprensión de su lógica propia y una construcción sobre los mismos.

De esta manera, se avanza hacia un pensamiento crítico y una auténtica apropiación de los derechos. Apropiación que se traduce en valores, percepciones, deseos y acciones hacia su defensa, revitalización, e incluso, su resignificación en medio de las relaciones sociales y prácticas al interior de la comunidad. El pensamiento crítico y la consiguiente transformación social se hacen evidentes y concretos en los espacios de su comunidad y de su vida familiar.

En el trabajo con y por los otros, encuentran respuesta a sus inquietudes existenciales y logran procesos de transformación no solo en su comunidad, sino en ellos mismos. En otras palabras, desde su trabajo en la comunidad, aportando, jalonando, promoviendo procesos en beneficio de sus vecinos, ellos mismos se van formando, adquieren saberes, percepciones y constituyen subjetividades frente a su existencia y a su relación con los otros. Desde esta perspectiva y a través de estos ejercicios, se van reconstruyendo los tejidos sociales en la medida en que se fortalecen los procesos de vinculación con lo comunal.

\section{El joven como sujeto político a partir de procesos de empoderamiento}

Los procesos de concienciación que se persiguen en los proyectos a través de la identificación y problematización crítica, logran develar la realidad y sus contradicciones. Son estos procesos de manifestación de las relaciones injustas y contradictorias, junto con los procesos en los que los participantes logran su reconocimiento como sujetos históricos en cuya acción persiguen la transformación de las estructuras y relaciones sociales en los que, con la progresiva constitución como sujetos sociales y políticos, se generan procesos de empoderamiento. "Una de las cosas más complicadas de uno como educador, como maestro, que uno se puede encontrar es cómo lograr que los estudiantes o sujetos que se están formando, piensen por sí mismos. Eso se Ilamaría empoderamiento; para que, pensándose por sí mismos, generen acciones propias" (E, párrafo 69). A través del reconocimiento de estas prácticas deshumanizantes y su contraste con las condiciones de vida digna protegidas por los derechos humanos, se pretende involucrar al sujeto con las problemáticas de su comunidad y emprender acciones hacia la superación de éstas.

Este empoderamiento que se persigue tras las prácticas de estos proyectos de formación, permite emerger otra categoría. Encontramos que en estas prácticas se reconoce al joven como un sujeto político que está en capacidad de emprender procesos de transformación y resignificación de las relaciones sociales al interior de las comunidades. 
En muchas comunidades, principalmente en aquellas localidades en las cuales se desarrollan estos proyectos, el joven es visto como un problema para el barrio. Fenómeno que termina excluyendo al joven de la vida en comunidad y favoreciendo el campo para el aumento de las problemáticas juveniles como el pandillismo, la delincuencia, la droga, la falta de oportunidades y espacios para los jóvenes. Estos proyectos intentan, por un lado, cambiar esta representación frente a los jóvenes y, por otro, comprometer a los jóvenes con dichos procesos. De esta manera, el joven inicia prácticas en torno a su comunidad y en torno a ellos mismos.

Es en esta labor en donde se fortalece la idea del joven como sujeto social y político que está en capacidad de organizarse y emprender acciones por su comunidad, a la vez que ellos mismos resignifican sus prácticas y reorientan sus proyectos de vida. Así, se van fortaleciendo en las prácticas mismas de estos proyectos procesos de empoderamiento en el reconocimiento del potencial del joven en la transformación de las relaciones sociales de su comunidad. Los jóvenes dan muestra de estas capacidades y de su papel activo en la reconstrucción de la sociedad, como sujetos críticos, históricos, sociales y políticos.

\section{Diálogo de saberes y reconocimiento del otro}

Hemos venido insistiendo en que un proyecto de formación en derechos humanos se inicia con aquello que es esencial y da origen a los derechos mismos: el reconocimiento del otro como un legítimo otro. Es decir, un otro con el cual comparto la naturaleza humana que nos otorga ciertas condiciones y calidad que denominamos dignidad. Dignidad que debe ser protegida pues en ella se circunscriben las condiciones mínimas que posibilitan nuestra realización como personas. En palabras de Freire, son las condiciones que permiten la realización ontológica de "ser más" bajo la óptica del ser humano como proyecto inacabado y en construcción.

La realización de este proyecto ontológico de humanización se inicia con la propia posibilidad de ser y pronunciar el mundo alejado de toda práctica autoritaria y domesticadora. El sujeto está en capacidad de construir su propia comprensión del mundo a partir de sus propias lecturas ${ }^{9}$.

9 Nos dice Freire en la Pedagogía del Oprimido: "Existir humanamente, es pronunciar el mundo, es modificarlo... la palabra no es privilegio de algunos hombres, sino derecho de todos los hombres" ( p. 97).
Y esto incluye las prácticas educativas. "El papel del educador o la educadora progresista que no puede ni debe omitirse, al proponer su 'lectura del mundo', es señalar que existen otras 'lecturas del mundo' diferentes de la suya y hasta antagónicas en ciertas ocasiones" (Freire, 2007, p. 95). Incluso, cuando se trata del propio discurso dentro de una práctica educativa alternativa.

El conocimiento es una construcción a partir del encuentro de dos sujetos con los conocimientos que cada uno ha podido construir desde su experiencia y encuentro con otros. La manera de hacer concreto y constructivo este encuentro es el diálogo ${ }^{10}$. Un diálogo donde ambos sujetos aportan, actúan y escuchan para interiorizar y relacionar sus saberes. El conocimiento no es posesión de nadie, es una construcción social. En consecuencia, el docente no puede transmitir conocimiento; transmite datos, información, experiencias pero no conocimiento, por la sencilla razón de que el conocimiento es un proceso continuo que necesita de la acción del sujeto, la investigación, la praxis para ser apropiado, lo cual le da vigencia y le permite superar el simple dato o información.

Es más, los conocimientos que traen los participantes a los encuentros son valorados profundamente por su riqueza y testimonios directos de la vida de la comunidad. Son lecturas, expresiones fieles que permiten radiografiar la realidad concreta del barrio, traer a la mesa de discusión esas problemáticas reales y existenciales, para reflexionarlas y repensarlas:

incluso, muchas veces, sus conocimientos son más importantes que una persona que ha desarrollado determinados grados académicos, porque ellos tienen una ventaja frente a esas personas, y es su vivencia diaria, su vivencia cotidiana. Y que desde esas cotidianidades es importante el construirse y el aportar esa misma experiencia en desarrollo de las comunidades (C1, párrafo 35)

Finalmente, después de analizar estos testimonios, es posible encontrar el correlato del "reconocimiento del otro" en los derechos humanos en el "diálogo de saberes" propuesto por Freire. Estos dos elementos se mezclan constantemente en las prácticas analizadas y se convierten en instrumentos que favorecen e impulsan los procesos y propósitos de los proyectos.

10 "El diálogo gana significado precisamente porque los sujetos dialógicos no solo conservan su identidad sino que la defienden y así crecen el uno con el otro. Por lo tanto el diálogo no nivela, no reduce el uno al otro" (Freire, 2007, p. 113). 


\section{Trascendencia del proceso de formación}

Estos proyectos nunca han perdido el nexo con lo concreto, con las realidades de las comunidades, con las problemáticas de los sujetos que asisten a los proyectos. En este sentido se han abordado las problemáticas desde los sentires, percepciones y proyecciones de los mismos participantes, quienes en sus búsquedas personales se han involucrado y comprometido con procesos y acciones por sus comunidades y por su entorno inmediato: sus familias, sus amigos, su cuadra, sus compañeros de clase.

De esta manera, podemos analizar el impacto de los proyectos en dos sentidos. A nivel personal son muy dicientes las palabras de don Pedro, líder de la fundación Codiepsir: "Eso es un poco como el justificar quién soy" (C1, párrafo 18). Con estas palabras cierra su relato respecto al proceso de formación del proyecto. Manifiesta así su constitución como sujeto político en tensión con su formación como sujeto social. La fundación es una respuesta a ese propósito de hacer algo por la comunidad y, especialmente, por los jóvenes, abriendo nuevos espacios de acción y expresión, nuevas oportunidades existenciales, nuevos sentidos a sus proyectos de vida que les ofreciesen a los jóvenes otras posibilidades diferentes al pandillismo y a la drogadicción. Su vida se define en este proyecto. Su acción por los otros justifica su existencia. Es su proyecto de vida y este sentir lo transmite a los participantes de la fundación. En la medida en que el individuo trabaja por la comunidad se construye como persona.

El empoderamiento que surge de la lectura crítica de la realidad y el proceso de concienciación, se hace efectivo en el fortalecimiento de las relaciones interpersonales de los individuos, en el solidarizarse con los problemas del otro, enterarse de su historia, sus dificultades y emprender procesos de apoyo para ayudar a salir de la dificultad y superar los problemas. Esto forma más que el sólo conocimiento, generalmente teórico, discursivo y abstracto. Es un verdadero proceso de constitución de subjetividades que no se queda en el simple reconocimiento ni en el enterarse de esas problemáticas, ni tampoco con el hecho de constatarlas en la realidad cercana, sino que se compromete, toca la existencia y moviliza hacia la acción por el otro, la opción fraternal por el otro, el hacerme responsable del otro. Estos son elementos fundamentales para una cultura de derechos humanos que se fortalece en esas subjetividades en torno al respeto y a la dignidad de las personas.

En otras palabras, el trabajo por la comunidad se convierte en instrumento pedagógico y terapéutico, que actúa sobre la persona misma e involucra su subjetividad y perspectivas frente a la vida y frente a sus mismas relaciones con los demás. De esta manera se resignifican sus relaciones con los otros, sus formas de acercarse a ellos, su manera de percibirlos, su manera de resolver los conflictos y diferencias, su manera de concebir al otro.

Estos proyectos logran un impacto tal a nivel personal y existencial de los jóvenes que fue posible para ellos el plantarse nuevos retos, nuevos rumbos para su vida a pesar de las dificultades del contexto en el que se encontraban y replantean su proyecto de vida lejos de las drogas y la delincuencia.

Respecto a lo que es la Corporación como tal, las evidencias de influencia en las personas están en los chicos que viviendo en esos sectores tan extremos hacen parte de las universidades públicas y privadas... la Corporación incide en la vida de unos chicos que, tal vez, si algún día no hubieran llegado al semillero de la Corporación, pues estarían relegados como muchos otros jóvenes se encuentran en nuestras comunidades (C2, párrafo 68).

Esta preparación y los nuevos elementos que consiguen forjar en su paso por universidades y diferentes actividades de formación, las revierten en el trabajo por su comunidad. Este compromiso nunca lo han olvidado.

\section{A manera de conclusión}

El siguiente cuadro nos permite identificar de manera concreta los cruces que permiten establecer la proximidad entre las pedagogías inspiradas en las obras de Freire y los proyectos de educación en derechos humanos.

Cuadro 1: Categorías teóricas: puntos de encuentro entre las pedagogías críticas y la educación en derechos humanos.

\section{Dentro de los derechos humanos}

Reconocimiento del otro.

Dignidad humana.

Transformación de las relaciones sociales.

Empoderamiento a través del reconocimiento de los derechos humanos.

Subjetividades en torno a los derechos humanos. 


\section{Desde las pedagogías críticas}

Pedagogía dialógica.

Alteridad.

Emancipación y Transformación social.

Pensamiento crítico, problematización y lectura crítica de la realidad.

Sujeto crítico-político.

Freire concibe la educación como práctica emancipadora partiendo de la concienciación promovida por la alfabetización. La educación es entendida como una acción cultural en la construcción de subjetividades aportando no solo datos e información, sino generando nuevas maneras de reflexionar, pensar, cuestionar e imaginar el mundo asumiendo nuevas codificaciones, actitudes y opciones frente a la realidad. Estos procesos de constitución de subjetividades se potencian dentro de una pedagogía emancipadora, lo cual no implica que dentro de la educación tradicional no se promuevan otras subjetividades.

La educación es un dispositivo, entre muchos otros, de intervención político-cultural. Si bien, Freire no es el único autor que permite acercarnos y pensar en una educación en derechos humanos con la intención de fortalecer el proceso de construcción de subjetividades, sí es un autor que en sus obras nos permite analizar varios elementos que enriquecen el proyecto y lo hacen viable, como lo hemos constatados en el estudio de las experiencias.

A su vez, la educación en derechos humanos permite, en primer lugar, que el sujeto reconozca sus derechos y valore la dignidad de ser humano. Este reconocimiento le permite concebir a los demás sujetos como auténticos seres humanos merecedores, por su dignidad, de condiciones de vida acordes con su naturaleza. El constatar que estas condiciones mínimas no se presentan por diversas razones e intereses, favorecen la conciencia crítica, sentimientos de rechazo, empoderamiento y alimentan deseos de cambio, de renovación, de transformación. Es, entonces, cuando la denuncia se convierte en dispositivo que propicia la memoria histórica y el compromiso por incidir en las relaciones y estructuras sociales ${ }^{11}$. Así se inician procesos de empoderamiento para la transformación a partir del reconocimiento de los derechos fundamentales.

11 "La utopía implica esa denuncia y ese anuncio, pero no permite que se agote la tensión entre ambos en torno a la producción del futuro antes anunciado y ahora un nuevo presente" (Freire, 2007, p. 87).
A través de este empoderamiento que fortalece la lectura crítica de la realidad y los procesos de concienciación se favorece, a su vez, la constitución de sujetos políticos que inciden en las dinámicas y decisiones en torno a las problemáticas de sus comunidades. Esto se hace evidente en las prácticas analizadas en donde los sujetos se ponen en la tarea de participar en las decisiones y procesos comunitarios, haciéndose escuchar y planteando alternativas o construyéndolas, según la situación. De esta manera, encuentran estos espacios de participación política como espacios propicios para, desde allí, trabajar por sus comunidades y por ellos mismos.

En resumen, es posible plantear un proyecto de formación en derechos humanos desde las pedagogías críticas por los elementos aquí mencionados. Estas prácticas se presentan como proyectos emancipadores y se evidencian en ellas procesos de constitución de subjetividades y sujetos sociales. De igual manera, comparten la formación de sujetos políticos promovida implícitamente y reafirman, en la clara perspectiva política de sus líderes asumida como proyecto de vida, la idea de que un proyecto político emancipador involucra la vida misma y exige coherencia. Finalmente, este punto de encuentro se hace más fuerte en la categoría de la pedagogía de la vida expresada en el diálogo cultural y el diálogo de saberes; fortalecida en el partir de la realidad concreta y sus problemáticas para su examen y reflexión; vivenciada en las prácticas que reconocen al sujeto con sus sentires, perspectivas y proyectos en el proceso de constitución de subjetividades y sujetos.

Ya para terminar, es necesario precisar otro hallazgo en esta investigación. No solo las pedagogías críticas enriquecen y viabilizan con sus aportes un proyecto de formación en derechos humanos que se proponga consolidar procesos de constitución de subjetividades en torno a una cultura de derechos humanos, sino que los derechos humanos también aportan a las pedagogías críticas. La educación en derechos humanos ofrece un nicho en el cual es posible hacer concretos los postulados de las pedagogías críticas. Estas experiencias en derechos permiten a las pedagogías críticas tener un campo de trabajo y de acción en el cual pueden pensarse, reformularse y realizarse como proyecto político y pedagógico. Los derechos humanos permiten espacios en los cuales las pedagogías críticas pueden avanzar en la consecución de estos proyectos. 
Defensoría del Pueblo. (2004). Red de Promotores de Derechos Humanos. ¿Qué son los derechos Humanos? Bogotá.

El Achkar, S. (2002). "Una mirada a la educación en derechos humanos desde el pensamiento de Paulo Freire. Prácticas de intervención político cultura". En: Estudios y otras prácticas intelectuales latinoamericanas en cultura y poder. Mato Daniel (compilador). Caracas-Venezuela: Consejo Latinoamericano de Ciencias Sociales, CLACSO.

Freire, P. (1971). La educación como práctica de la libertad. Montevideo-Uruguay: Editorial Tierra Nueva.

Freire, P. (1994). La naturaleza política de la educación. BarcelonaEspaña: Editorial Planeta-Agostini.

Freire, P. (1997). Pedagogía de la autonomía. México: Editorial Siglo XXI.

Freire, P. (2007). Pedagogía de la esperanza. México: Editorial Siglo XXI. Séptima Edición.

Freire, P. Pedagogía del Oprimido. Bogotá: Editorial América Latina.

Magendzo, A. (2005). Educación en derechos humanos. Bogotá: Editorial Magisterio.

Torres, A. (2007). La educación popular. Trayectoria y actualidad. Bogotá: Editorial El Búho.

Torres, A. (2006). "Subjetividad y sujeto: Perspectiva para abordar lo social y lo educativo". En: Revista Colombiana de Educación, N ${ }^{\circ} 50$, pp. 87-104.

Zemelman, H. (2006). "Ensayo Pensar la sociedad y a los sujetos sociales". En: Revista Colombiana de Educación, Nº 50, pp. 15-34.

\section{Diálogo del conocimiento}

El artículo del profesor Espinel que acabamos de leer, abre sin duda un espacio de reflexión-acción a propósito de la formación en derechos humanos desde la perspectiva de las pedagogías críticas, no sólo porque acude a los planteamientos de Paulo Freire, sino porque reconoce la importancia de la formación en derechos humanos desde una perspectiva de intervención de los sujetos partícipes de los procesos de formación como sujetos actuantes, en el sentido de considerarse sujetos contextuales, dialógicos y políticos.

Los derechos humanos no pueden ser vistos simplemente como axiomas que todos debemos conocer o aprender, se trata de considerarlos indispensables para la vida cotidiana, en particular bajo las condiciones actuales de nuestro país, donde se requiere de la tramitación de los conflictos a partir de procesos de reconocimiento de los otros, de la valoración de los sujetos, del reconocimiento, así como de la defensa de las experiencias colectivas, conocimientos particulares de personas y comunidades en condiciones de desigualdad, violencia e inequidad; en ese sentido, las acciones de formación en derechos humanos guiadas por una perspectiva crítica de la pedagogía, hacen de los escenarios propios, elementos articuladores de una reflexión y elaboración de propuestas por la defensa de los derechos humanos.

El profesor Espinel muestra cómo es posible crear espacios de formación que llevan a los sujetos del mismo proceso, a reflexiones que trasciendan la mera información y se conviertan en los articuladores de las relaciones sociales, permitiendo el empoderamiento de los sujetos y sus comunidades en pro de las transformaciones sociales en clave de emancipación, lo que ubica su trabajo en las dimensiones articuladoras de las pedagogías críticas y evidencia la necesidad de que la pedagogía sea cada vez más política y la política cada vez más pedagógica como ya lo enunciara Giroux. Todo ejercicio de formación sobre asuntos ético-políticos debe estar atravesado por un enfoque pedagógico que se encuentre en consonancia con los mismos, haciendo de la formación una vivencia que trasciende y transforma. No tiene sentido enseñar los derechos humanos si no se forma en los derechos humanos, ya que de la primera forma no queda sino la letra muerta en el papel; los derechos, como la constitución, deben vivirse más que aprenderse, lo que sugiere que la razón debe llenarse de motivos para la acción y no necesariamente ocurre teniendo la información.

Los derechos deben ser una vivencia permanente y si no es así, deben ser el motor que impulse la exigencia individual y colectiva de que así sea.

Se valora la importancia de esta reflexión porque reconoce una práctica pedagógica de organizaciones sociales en contextos de exclusión y de violación de los derechos humanos así como de desconocimiento de los mismos, visibiliza y empodera la experiencia como ejercicio ético-político-pedagógico; sin embargo, aún nos deja algunas preguntas que como maestros hacemos de manera obligada: ¿Cómo se realiza la formación por parte de estas organizaciones sociales en contextos tan complejos? ¿Cuál es la población que interactúa en estos escenarios? ¿Desde qué perspectiva se asume la promoción y defensa de los derechos humanos? ¿Qué diferencias y similitudes se pueden observar en cuanto a los procesos de formación en escenarios distintos dentro de las mismas comunidades? Comprendiendo por supuesto, que el artículo abre un espacio importante para las voces de los agentes del proceso de formación e intenta ir más allá de la mera descripción de la experiencia aventurándose en la consolidación de una reflexión político-pedagógica bastante sugerente. 